\title{
Distribuição vertical e diversidade de coleópteros em povoamento de Pinus taeda L. (Pinaceae)
}

\author{
Mateus Alves Saldanha ${ }^{1 *}$, Ervandil Corrêa Costa ${ }^{2}$, Leonardo Mortari Machado ${ }^{3}$, Dayanna do Nascimento \\ Machado $^{4}$, Jéssica Maus da Silva ${ }^{5}$, Leandra Pedron ${ }^{6}$, Camila Fonseca Galvan ${ }^{7}$, Bruna Casanova Silva ${ }^{8}$
}

DOI: https://doi.org/10.35699/2447-6218.2020.21654

\begin{abstract}
Resumo:
O padrão de voo dos insetos pode ser considerado um importante fator biológico no manejo de potenciais insetos-praga. Deste modo, este trabalho objetivou verificar a altura preferencial de voo dos coleópteros associados a um plantio de Pinus taeda L pertencente ao Centro de Pesquisa em Florestas, em Santa Maria, Rio Grande do Sul. Para isto, foram instaladas armadilhas de interceptação de voo distribuídas nas alturas de 0,$5 ; 1,0 ; 1,5 ; 2,0 ; 2,5 ; 3,0 ; 3,5$; 4,0; 4,5; 5,0; 5,5 e 6,0 metros acima da superfície do solo, com três repetições e com um espaço de 30 metros entre as repetições. Foram coletados 570 indivíduos, distribuídos em 34 famílias e 116 espécies, com o acme populacional no início de novembro de 2011. As famílias Cerambycidae, Curculionidae, Trogossitidae e Scarabaeidae apresentaram a maior abundância. Euetheola humilis (Burmeister, 1847) destacou-se, com 42 indivíduos amostrados no período de novembro de 2011 a maio de 2012, predominantemente, nas alturas de 3,5 m, 4,5 m e 5,0 m. Não foram encontradas diferenças estatísticas entre as diferentes alturas e a abundância total de coleópteros, demonstrando que, neste estudo, os insetos da Ordem Coleoptera não possuem preferência por uma altura de voo em povoamento de Pinus taeda.
\end{abstract}

Palavras-chave: Armadilha de interceptação de voo. Monitoramento de insetos. Padrão de voo.

\section{Vertical distribution and diversity of beetles in a stand of Pinus taeda L. (Pinaceae)}

\begin{abstract}
:
The flight pattern of insects can be considered an important biological factor in the management of potential pest insects. Thus, this study aimed to verify the preferred flight height of the coleoptera associated with a plantation of Pinus taeda L. belonging to the Forest Research Center, in Santa Maria, Rio Grande do Sul. For this purpose, distributed flight interception traps were installe,corresponding to heights of $0.5 ; 1.0 ; 1.5 ; 2.0 ; 2.5 ; 3.0 ; 3.5 ; 4.0 ; 4.5 ; 5.0 ; 5.5$ and 6.0 meters above the soil surface, with three repetitions and with an interval of 30 meters between repetitions. 570 individuals were collected, distributed in 34 families and 116 species, with the acme population occurring in the
\end{abstract}

${ }^{1}$ Universidade Federal de Santa Maria. Centro de Ciências Rurais. Departamento de Defesa Fitossanitária. Santa Maria, RS. Brasil. https://orcid.org/0000-0002-1788-8179.

${ }^{2}$ Universidade Federal de Santa Maria. Centro de Ciências Rurais. Departamento de Defesa Fitossanitária. Santa Maria, RS. Brasil. https://orcid.org/0000-0001-7348-8826.

${ }^{3}$ Universidade do Estado de Santa Catarina. Centro de Ciências Agroveterinárias. Lages, SC. Brasil.

http://orcid.org/0000-0001-9824-8087.

${ }^{4}$ Universidade Federal de Santa Maria. Centro de Ciências Rurais. Departamento de Defesa Fitossanitária. Santa Maria, RS. Brasil. https://orcid.org/0000-0001-9837-5369.

${ }^{5}$ Universidade Federal de Santa Maria. Centro de Ciências Rurais. Departamento de Defesa Fitossanitária. Santa Maria, RS. Brasil. https://orcid.org/0000-0003-4564-0389.

${ }^{6}$ Universidade Federal de Santa Maria. Centro de Ciências Rurais. Departamento de Defesa Fitossanitária. Santa Maria, RS. Brasil. https://orcid.org/0000-0002-0712-241X.

${ }^{7}$ Universidade Federal de Santa Maria. Centro de Ciências Rurais. Departamento de Defesa Fitossanitária. Santa Maria, RS. Brasil. https://orcid.org/0000-0002-2995-3038.

${ }^{8}$ Universidade Federal de Santa Maria. Centro de Ciências Rurais. Departamento de Defesa Fitossanitária. Santa Maria, RS. Brasil. https://orcid.org/0000-0002-7524-8479.

*Autor para correspondência: mtsmateusalves@gmail.com

Recebido para publicação em 01 de junho de 2020. Aceito para publicação em 19 de outubro de 2020.

e-ISSN: 2447-6218 / ISSN: 2447-6218. Atribuição CC BY. 
beginning of November 2011. The families Cerambycidae, Curculionidae, Trogossitidae and Scarabaeidae presented the greatest abundance. Euetheola humilis (Burmeister, 1847) stood out, with 42 individuals sampled during the collection period (November 2011 to May 2012), predominantly, at heights of $3.5 \mathrm{~m}, 4.5 \mathrm{~m}$ and $5.0 \mathrm{~m}$. No statistical differences were found between the different heights in the flight interception traps and the total abundance of beetles, demonstrating that the insects of the Coleoptera Order do not have a preference for flight height in P. taeda stands.

Key words: Flight pattern. Insect monitoring. Flight interception trap.

\section{Introdução}

No Brasil, as plantações florestais ocupam 7,84 milhões de ha sendo responsáveis por mais de $90 \%$ de toda a madeira utilizada para fins produtivos, ainda contribuem para a conservação da biodiversidade, recuperação de áreas degradadas, preservação do solo, regulação dos recursos hídricos e geração de energia renovável (IBÁ, 2019). As espécies de Pinus se destacam por ocupar 1,6 milhão de ha, e os Estados da região Sul do Brasil apresentam as melhores condições edafoclimáticas para o estabelecimento e desenvolvimento desse gênero (IBÁ, 2019).

No entanto, o constante incremento de áreas com plantios homogêneos, de espécies exóticas de rápido crescimento, houve também um acréscimo significativo nos problemas fitossanitários, especialmente com insetos (Machado; Costa, 2017). Conforme relatado por Flechtmann et al. (1995) estes insetos podem limitar o crescimento, o desenvolvimento e a reprodução das árvores e por serem potenciais vetores de doenças, bactérias, fungos e vírus (Machado, 2013).

Dentre as pragas prejudiciais às essências florestais, destacam-se aquelas conhecidas como coleobrocas, cujos danos são importantes em algumas espécies. Pode-se citar como exemplo espécies da família Curculionidae, que dentre os insetos monitorados em povoamentos florestais, são os mais relevantes, pois proporcionam danos expressivos na madeira de toras recém-abatidas quando estão expostas no campo (Carvalho, Trevisan; 2015). Tem-se também os cerambicídeos, que são consideradas pragas relevantes na Entomologia Florestal, conhecidas como serradores ou aneladores pelo grande número de plantas hospedeiras e pelos danos que causam em determinadas essências florestais, quando cultivadas em florestas homogêneas (Bernardi et al., 2010, Lemes et al., 2011). De acordo com Bertim (2013), os coleópteros adaptam-se facilmente aos diferentes ambientes florestais, assim o monitoramento se torna a forma mais eficaz contra os surtos.

O padrão de voo dos insetos pode ser considerado um importante fator biológico no manejo de potenciais insetos-praga, pois a altura de voo varia entre as espécies e demonstra estar relacionada com o ponto em que ocorre infestação na planta hospedeira. Algumas espécies capturadas próximas ao nível do solo, geralmente atacam a parte inferior do tronco, por exemplo (Machado; Costa, 2017). O padrão de voo também está principalmente relacionado a reprodução (acasalamento) e à obtenção de alimento (Bertim, 2013).
Neste contexto, o estudo da altura de voo dos insetos possibilita avaliar a distribuição vertical das possíveis injúrias, sendo utilizado na definição de uma ferramenta de monitoramento, auxiliando na adoção de medidas preventivas para o manejo de insetos-praga (Peres Filho et al., 2012; Bertim, 2013). Este trabalho tem por objetivo realizar um levantamento qualitativo (espécies) e quantitativo (abundância) dos coleópteros presentes em um povoamento de Pinus taeda L., caracterizando e delimitando a altura preferencial de voo das principais espécies associadas ao povoamento.

\section{Material e métodos}

\section{Caracterização da área de estudo}

O presente trabalho foi conduzido no período de novembro de 2011 a maio de 2012 , em povoamento de $P$. taeda com aproximadamente 25 anos de idade, em uma área de 2 ha (2940’5.79" S e 5355'16.60" O), pertencente ao Centro de Pesquisa em Florestas, Santa Maria, Rio Grande do Sul.

O povoamento apresentava um espaçamento variável (entre 1 e $5 \mathrm{~m}$ ). Essa variação no espaçamento se deve ao fato de a área ser uma condução da regeneração natural, a qual foi manejada nos primeiros anos de idade para formar um povoamento proveniente de regeneração. A altura média das árvores foi estimada em 18,35 m, com um diâmetro médio de $25,3 \mathrm{~cm}$ e uma altura média do dossel de 14 m (Machado, 2013).

O clima da região é subtropical úmido oceânico, sem estação seca e com verão quente; a temperatura média mensal é de $19^{\circ} \mathrm{C}$, sendo a média dos meses mais quentes superior a $30^{\circ} \mathrm{C}$ e dos meses mais frios entre 13 e $18{ }^{\circ} \mathrm{C}$, com precipitação média anual de $1.770 \mathrm{~mm}$, com média anual de 113 dias de chuva (ALVARES et al., 2017).

\section{Armadilha de interceptação de voo e procedimento de captura}

O modelo de armadilha etanólica de impacto utilizado no presente estudo foi desenvolvido por Murari et al. (2012) e intitulado PET-SM, sendo que tal armadilha era de conhecimento dos autores, porém, só foi publicada em 2012, mesmo o trabalho do presente estudo ter iniciado em 2011. Em novembro de 2011 três árvores do povoamento de $P$. taeda foram selecionadas aleatoriamente para a instalação do conjunto de armadilhas. Cada árvore estava distante em pelo menos 30 metros entre 
Distribuição vertical e diversidade de coleópteros em povoamento de Pinus taeda L. (Pinaceae)

si e aproximadamente 50 metros da bordadura. Cada conjunto de armadilha estava composto por 12 armadilhas que foram alinhadas verticalmente totalizando seis metros de altura e penduradas nas árvores selecionadas. As armadilhas foram dispostas nas seguintes alturas: 0,5 ; 1,$0 ; 1,5 ; 2,0 ; 2,5 ; 3,0 ; 3,5 ; 4,0 ; 4,5 ; 5,0 ; 5,5$ e 6,0 metros acima da superfície do solo. Foram realizadas 13 coletas, sendo que cada conjunto de armadilha permanecia na área por um período de 15 dias.

\section{Triagem e identificação dos espécimes}

Após a coleta, os insetos foram transportados ao Laboratório de Pesquisa em Entomologia Florestal (CCR/UFSM), onde foi realizada a triagem do material, tendo por base as características morfológicas dos insetos, com auxílio de lupa binocular, pinça e pincel. Após a morfotipagem os espécimenes foram alocados em tubos plásticos do tipo Eppendorf de 1,5 mL, contendo álcool 70 \%, sendo posteriormente enviado ao Dr. Pedro Giovâni da Silva, do Laboratório de Ecologia de Insetos, Instituto de Ciências Biológicas da Universidade Federal de Minas Gerais, para identificação das espécies de coleópteros. Para esse estudo levou-se em consideração os dados das diferentes famílias de Coleoptera, com exceção de Scolytidae, pois os mesmos já haviam sido avaliados por Machado e Costa (2017).

\section{Análise estatística}

Os parâmetros ecológicos Riqueza (S), Abundância (N) e Diversidade de Shannon (H') foram determinados com o auxílio do software Past versão 2.17 c (Hammer; Harper; Ryan, 2001). Para verificar a faixa preferencial de voo dos coleópteros, assim como a flutuação populacional dos indivíduos, foram realizadas análises gráficas utilizando o Microsoft Excel versão 2019, a partir de análise de regressão polinomial de modelo quadrático. Para análise da distribuição vertical foram consideradas as espécies mais abundantes.

\section{Resultados e discussão}

\section{Espécies identificadas e flutuação populacional}

Os 570 espécimes coletados no povoamento de $P$. taeda foram distribuídos em 116 morfoespécies, com 42 identificadas em nível de espécie e 74 em gênero (Tabela 1).

Tabela 1 - Riqueza (S) por família, abundância $(\mathrm{N})$ e frequência relativa (\%) de coleópteros coletados com armadilha de interceptação de voo, em alturas de 0,5 a 6,0 m, em povoamento de Pinus taeda, entre novembro de 2011 e maio de 2012, no município de Santa Maria/RS.

\begin{tabular}{|c|c|c|c|}
\hline Famílias & Gênero/Espécies & $\mathbf{N}$ & $\%$ \\
\hline \multirow{4}{*}{ Alleculidae $(S=4)$} & Lobopoda sp.1 & 3 & 0,53 \\
\hline & Lobopoda sp.2 & 10 & 1,75 \\
\hline & Lobopoda sp.3 & 2 & 0,35 \\
\hline & Lobopoda sp.4 & 3 & 0,53 \\
\hline \multirow[t]{2}{*}{ Anobiidae $(S=1)$} & Dasytanobium inaequale (Pic, 1902) & 5 & 0,88 \\
\hline & Domoptolis menestriesii (Boheman, 1845) & 11 & 1,93 \\
\hline \multirow[t]{3}{*}{ Anthribidae $(S=3)$} & Eugonus subcylindricus (Fahraeus, 1839) & 6 & 1,05 \\
\hline & Toxonotus sp. & 5 & 0,88 \\
\hline & Bostrychopsis uncinata (Germar, 1824) & 8 & 1,40 \\
\hline \multirow[t]{2}{*}{ Bostrichidae $(\mathrm{S}=3)$} & Melalgus sp. & 1 & 0,18 \\
\hline & Xyloprista sp. & 2 & 0,35 \\
\hline \multirow[t]{2}{*}{ Bothrideridae $(S=1)$} & Bothrideres sp. & 1 & 0,18 \\
\hline & Chauliognathus flavipes (Fabricius, 1781) & 2 & 0,35 \\
\hline \multirow[t]{3}{*}{ Cantharidae $(S=2)$} & Discodon sp.1 & 1 & 0,18 \\
\hline & Discodon sp.2 & 1 & 0,18 \\
\hline & Lebia sp. & 4 & 0,70 \\
\hline \multirow[t]{2}{*}{ Carabidae $(S=3)$} & Cenothyla sp. & 1 & 0,18 \\
\hline & Loxandrus sp. & 3 & 0,53 \\
\hline
\end{tabular}


Saldanha, M. A. et al.

\begin{tabular}{|c|c|c|c|}
\hline Famílias & Gênero/Espécies & $\mathbf{N}$ & $\%$ \\
\hline \multirow{26}{*}{ Cerambycidae $(S=26)$} & Cosmotoma viridana (Lacordaire, 1872) & 6 & 1,05 \\
\hline & Ateralphus dejeani (Lane, 1973) & 4 & 0,70 \\
\hline & Callia paraguaya (Galileo \& Martins, 1990) & 4 & 0,70 \\
\hline & Chariergus tabidus (Klug, 1825) & 4 & 0,70 \\
\hline & Chlorida costata (Serville, 1834) & 3 & 0,53 \\
\hline & Chydarteres taeniatus (Germar, 1824) & 2 & 0,35 \\
\hline & Colobothea sp. & 2 & 0,35 \\
\hline & Cotyclytus curvatus (Germar, 1821) & 10 & 1,75 \\
\hline & Desmiphora cucullata (Thomson, 1868) & 3 & 0,53 \\
\hline & Desmiphora intonsa (Germar, 1824) & 2 & 0,35 \\
\hline & Eburodacrys sexguttata (Lameere, 1884) & 2 & 0,35 \\
\hline & Eryphus bipunctatus (Perty, 1832) & 3 & 0,53 \\
\hline & Estola sp. & 3 & 0,53 \\
\hline & Eutrypanus dorsalis (Germar, 1824) & 2 & 0,35 \\
\hline & Lophopoeum carinatulum (Bates, 1863) & 7 & 1,23 \\
\hline & Martinsellus signatus (Gyllenhal, 1817) & 5 & 0,88 \\
\hline & Mecometopus bicinctus (Aurivillius, 1920) & 8 & 1,40 \\
\hline & Megacyllene falsa (Chevrolat, 1862) & 2 & 0,35 \\
\hline & Neoclytus pusillus (Laporte \& Gory, 1835) & 12 & 2,11 \\
\hline & Neoclytus ypsilon (Chevrolat, 1862) & 4 & 0,70 \\
\hline & Neocorus ibidionoides (Serville, 1834) & 6 & 1,05 \\
\hline & Nyssodrysina lignaria (Bates, 1864) & 10 & 1,75 \\
\hline & Paromoeocerus barbicornis (Fabricius, 1792) & 4 & 0,70 \\
\hline & Protosphaerion variabile (Gounelle, 1909) & 5 & 0,88 \\
\hline & Stultutragus poecilus (Bates, 1873) & 6 & 1,05 \\
\hline & Tilloglomus spectabilis (Martins, 1975) & 4 & 0,70 \\
\hline Chelonariidae $(S=1)$ & Chelonarium sp. & 3 & 0,53 \\
\hline \multirow{2}{*}{ Chrysomelidae ( $\mathrm{S}=2$ ) } & Colaspis sp. & 2 & 0,35 \\
\hline & Stilodes sp. & 3 & 0,53 \\
\hline \multirow{3}{*}{ Cleridae $(S=3)$} & Corinthiscus sp.1 & 23 & 4,04 \\
\hline & Corinthiscus sp. 2 & 2 & 0,35 \\
\hline & Enoclerus sp. & 4 & 0,70 \\
\hline \multirow{2}{*}{ Coccinellidae $(S=2)$} & Cycloneda sanguinea (Linnaeus, 1763) & 5 & 0,88 \\
\hline & Harmonia axyridis (Pallas, 1773) & 4 & 0,70 \\
\hline Cucujidae $(S=1)$ & Scalidia sp. & 2 & 0,35 \\
\hline
\end{tabular}


Distribuição vertical e diversidade de coleópteros em povoamento de Pinus taeda L. (Pinaceae)

\begin{tabular}{|c|c|c|c|}
\hline Famílias & Gênero/Espécies & $\mathbf{N}$ & $\%$ \\
\hline \multirow{11}{*}{ Curculionidae $(S=11)$} & Archocopterus sp. & 3 & 0,53 \\
\hline & Airosimus jacobi (Hustache, 1938) & 4 & 0,70 \\
\hline & Apocnemidophorus sp. & 4 & 0,70 \\
\hline & Eurycorynes excavatus (Wollaston, 1873) & 4 & 0,70 \\
\hline & Eurycorynes sp. & 5 & 0,88 \\
\hline & Heilus sp.1 & 2 & 0,35 \\
\hline & Heilus sp.2 & 3 & 0,53 \\
\hline & Macrocopturus sp. & 5 & 0,88 \\
\hline & Naupactus sp.1 & 2 & 0,35 \\
\hline & Naupactus sp.2 & 3 & 0,53 \\
\hline & Pantomorus sp. & 3 & 0,53 \\
\hline \multirow{8}{*}{ Elateridae $(S=8)$} & Aeolus sp. & 1 & 0,18 \\
\hline & Conoderus sp.1 & 4 & 0,70 \\
\hline & Conoderus sp.2 & 3 & 0,53 \\
\hline & Conoderus sp.3 & 3 & 0,53 \\
\hline & Conoderus sp.4 & 3 & 0,53 \\
\hline & Crepidius sp. & 2 & 0,35 \\
\hline & Pherhimius fascicularis (Fabricius, 1787) & 2 & 0,35 \\
\hline & Probothrium sp. & 3 & 0,53 \\
\hline \multirow{3}{*}{ Erotylidae $(S=3)$} & Gibbifer adrianae (Alvarenga, 1976) & 3 & 0,53 \\
\hline & Hapalips sp. & 1 & 0,18 \\
\hline & Iphiclus sp. & 2 & 0,35 \\
\hline Eucnemidae $(\mathrm{S}=1)$ & Gastraulacus sp. & 3 & 0,53 \\
\hline Histeridae $(S=1)$ & Hister sp. & 3 & 0,53 \\
\hline \multirow{2}{*}{ Hybosoridae $(S=2)$} & Chaetodus exaratus (Arrow, 1909) & 3 & 0,53 \\
\hline & Germarostes metallicus (Harold, 1874) & 5 & 0,88 \\
\hline Hydrophilidae $(S=1)$ & Berosus sp. & 12 & 2,11 \\
\hline Lucanidae $(S=1)$ & Zikanius sp. & 6 & 1,05 \\
\hline \multirow{2}{*}{ Melandryidae $(S=2)$} & Eudircaea lticornis (Champion, 1916) & 7 & 1,23 \\
\hline & Phloiotrya sp. & 5 & 0,88 \\
\hline Melolonthidae $(\mathrm{S}=1)$ & Plectris sp. & 2 & 0,35 \\
\hline \multirow{5}{*}{ Mordellidae $(S=5)$} & Mordella sp.1 & 1 & 0,18 \\
\hline & Mordella sp.2 & 3 & 0,53 \\
\hline & Mordella sp.3 & 2 & 0,35 \\
\hline & Mordella sp.4 & 1 & 0,18 \\
\hline & Tomoxia sp. & 4 & 0,70 \\
\hline
\end{tabular}


Saldanha, M. A. et al.

\begin{tabular}{|c|c|c|c|}
\hline Famílias & Gênero/Espécies & $\mathbf{N}$ & $\%$ \\
\hline \multirow{5}{*}{ Nitidulidae $(S=5)$} & Camptodes sp. & 4 & 0,70 \\
\hline & Colopterus sp.1 & 1 & 0,18 \\
\hline & Colopterus sp.2 & 1 & 0,18 \\
\hline & Colopterus sp. 3 & 4 & 0,70 \\
\hline & Lobiopa sp. & 4 & 0,70 \\
\hline Oedemeridae $(S=1)$ & Copidita sp. & 5 & 0,88 \\
\hline Platypodidae $(S=1)$ & Platypus sulcatus (Chapuis, 1865) & 8 & 1,40 \\
\hline \multirow[t]{4}{*}{ Ptilodactylidae $(\mathrm{S}=1)$} & Ptilodactyla sp. & 6 & 1,05 \\
\hline & Euetheola humilis (Burmeister, 1847) & 42 & 7,37 \\
\hline & Ateuchus sp. & 1 & 0,18 \\
\hline & Canthon sp. & 2 & 0,35 \\
\hline \multirow[t]{4}{*}{ Scarabaeidae $(S=7)$} & Isonychus sp. & 2 & 0,35 \\
\hline & Ontherus sp. & 2 & 0,35 \\
\hline & Onthophagus hircus (Billberg, 1815) & 4 & 0,70 \\
\hline & Uroxys dilaticollis (Blanchard, 1845) & 4 & 0,70 \\
\hline Scirtidae $(S=1)$ & Cyphon sp. & 3 & 0,53 \\
\hline Staphylinidae $(\mathrm{S}=1)$ & Quedius sp. & 4 & 0,70 \\
\hline \multirow{2}{*}{ Tenebrionidae $(S=2)$} & Acropteron sp. & 2 & 0,35 \\
\hline & Platydema sp. & 2 & 0,35 \\
\hline Tetratomidae $(S=1)$ & Eustrophinus sp. & 7 & 1,23 \\
\hline \multirow{7}{*}{ Trogossitidae $(S=7)$} & Temnoscheila sp.1 & 13 & 2,28 \\
\hline & Temnoscheila sp.2 & 34 & 5,96 \\
\hline & Temnoscheila sp.3 & 5 & 0,88 \\
\hline & Temnoscheila sp.4 & 3 & 0,53 \\
\hline & Tenebroides sp.1 & 22 & 3,86 \\
\hline & Tenebroides sp.2 & 22 & 3,86 \\
\hline & Tenebroides sp. 3 & 1 & 0,18 \\
\hline Total geral & & 570 & 100 \\
\hline
\end{tabular}

As famílias mais abundantes foram Cerambycidae, Trogossitidae, Scarabaeidae e Curculionidae com 123, 100, 57 e 38 espécimes, respectivamente, representando $55,8 \%$ do total coletado (Tabela 2 ). Este resultado corrobora com o estudo realizado por Ganho e Marinoni (2006) que, utilizando armadilhas etanólicas em plantação de Pinus elliottii na região Sul do Brasil, verificaram que a família Cerambycidae foi a mais abundante. Possivelmen- te este resultado está relacionado ao fato de que estes insetos são atraídos pelo álcool e por árvores estressadas ou em senescência. Bernardi et al. (2010) encontraram em maior abundância as famílias Cerambycidae e Scarabeidae, coletados com armadilhas etanólicas e luminosas em plantio de Eucalyptus spp., sendo estes métodos de coleta importantes ferramentas para o monitoramento de espécies dessas famílias em povoamentos florestais. 
Distribuição vertical e diversidade de coleópteros em povoamento de Pinus taeda L. (Pinaceae)

Tabela 2 - Famílias de Coleoptera mais abundantes coletadas com armadilha de interceptação de voo, em alturas de 0,5 a 6,0 m, em povoamento de Pinus taeda, entre novembro de 2011 e maio de 2012, no município de Santa Maria/RS.

\begin{tabular}{ccccc}
\hline \multirow{2}{*}{ Alturas $(\mathbf{m})$} & \multicolumn{3}{c}{ Famílias } \\
\cline { 2 - 5 } 0,5 & Cerambycidae & Curculionidae & Scarabaeidae & Trogossitidae \\
\hline 1,0 & 9 & 3 & 3 & 9 \\
1,5 & 16 & 4 & 2 & 12 \\
2,0 & 7 & 7 & 5 & 9 \\
2,5 & 12 & 5 & 3 & 10 \\
3,0 & 13 & 1 & 6 & 9 \\
3,5 & 2 & 1 & 10 & 11 \\
4,0 & 9 & 7 & 2 & 4 \\
4,5 & 13 & 1 & 5 & 7 \\
5,0 & 13 & 1 & 6 & 8 \\
5,5 & 16 & 6 & 5 & 8 \\
6,0 & 6 & 2 & 4 & 6 \\
\hline Total & 7 & 0 & 6 & 7 \\
\hline
\end{tabular}

As famílias Trogossitidae e Curculionidae apresentaram a segunda e terceira maior riqueza entre as famílias encontradas (100 e 38 espécimes, respectivamente). Tal resultado não foi observado em estudos realizados em plantio de Eucalyptus grandis (Freitas et al., 2002), onde foram observadas as famílias Cerambycidae e Sacarabeidae em maior abundância. Já em plantio de Eucalyptus urophylla (Pinto et al., 2004) verificaram a família Carabidae como sendo a mais abundante em seu levantamento. Isso pode ser explicado em função das diferentes localidades em que os estudos foram conduzidos, pois a incidência de insetos está relacionada às condições edafoclimáticas do meio. Além disto as diferentes espécies florestais estudadas proporcionam o estabelecimento de entomofauna específica em cada local.

Embora nenhuma das espécies tenha sido amplamente amostrada, os cerambicídeos apresentaram a maior diversidade de espécies. Fato semelhante ocorreu no trabalho realizado por Dorval et al. (2007) em povoamentos de E. citriodora e E. urophylla, onde utilizando armadilhas etanólicas instaladas a 1,5 $\mathrm{m}$ da superfície do solo, coletou várias espécies de Cerambycidae em baixa abundância.

Dentre as diversas espécies de Cerambycidae, as pertencentes ao gênero Neoclytus somaram 17, $89 \%$ do total de indivíduos dessa família. Neoclytus pussilus (Laporte \& Gory, 1835) é citada no Brasil por Zanuncio et al. (1993), como potente broqueadora de troncos de Eucalyptus pellita, Eucalyptus tereticornis e Eucalyptus urophylla (Myrtaceae). O mesmo autor afirma que insetos do gênero Neoclytus possuem alta capacidade de dano em diversas espécies florestais. os quais se caracterizam pela abertura de galerias no tronco das árvores, como observado por Peres Filho et al. (2006), que relatam a presença de $N$. pusillus em toras de Tectona grandis (Lamiaceae) estocadas por 30 dias, apresentando inúmeras galerias subcorticais com larvas de primeiro instar.

A família Scarabaeidae possui a espécie com maior abundância, Euetheola humilis (Burmeister, 1847) com 42 indivíduos, representando 7,36\% do total. Corroborando com o resultado obtido nesse trabalho, Bernardi et al. (2010) coletaram em armadilhas luminosas e etanólicas E. humilis como espécie mais abundante, representando 21,5\% dos coleópteros coletados em plantio de Eucalyptus spp., na região sul do Rio Grande do Sul. Diante disso, e levando em conta a afirmação de Móron (2004), de que a maior parte das espécies de insetos edafícolas deve estar se adaptando às condições de ecótonos e de agroecossistemas de cultivo, E. humilis pode se estabelecer como praga da pinus, visto que já se tem relato desta espécie atacando Eucalyptus saligna Smith no Sul do Rio Grande do Sul (Bernardi et al., 2008). Assim, esta espécie deve ter seus danos e população monitorada nos próximos anos para que se possa ter uma melhor avaliação de sua importância para a cultura do pinus.

Relação entre a abundância e a riqueza total em função da altura de voo

Não foi identificada relação entre a abundância total dos coleópteros estudados e as diferentes alturas de voo 
em que as armadilhas foram instaladas no povoamento de $P$. taeda (Figura 1), pois a equação gerada apresentou um baixo coeficiente de determinação entre a abundância total e as diferentes alturas de voo $\left(\mathrm{R}^{2}=0,0802\right)$. Dessa maneira, o total de coleópteros coletados não apresentou padrão de voo em nenhuma das alturas estudadas (de 0,5 a 6,0 metros acima da superfície do solo).

Figura 1 - Abundância total de coleópteros coletados com armadilha de interceptação de voo, em diferentes alturas, em povoamento de $P$. taeda, entre novembro de 2011 e maio de 2012, no município de Santa Maria/RS.

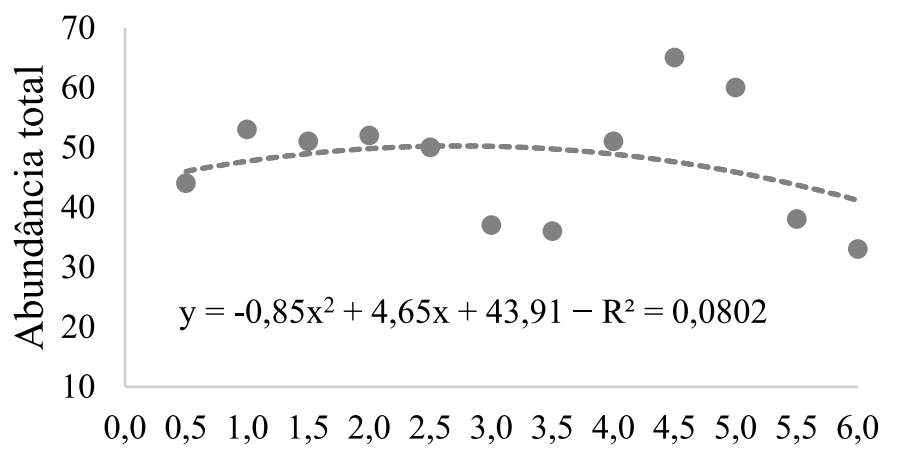

Alturas (m)

Também não foi identificada relação entre a riqueza total dos coleópteros estudados e as diferentes alturas de voo que as armadilhas foram instaladas no povoamento de $P$. taeda (Figura 2), pois a equação gerada também apresentou um baixo coeficiente de de- terminação entre a riqueza total e as diferentes alturas de voo $\left(R^{2}=0,0209\right)$. Dessa maneira, a diversidade total dos coleópteros coletados não apresentou padrão de voo em nenhuma das alturas estudadas (de 0,5 a 6,0 metros acima da superfície do solo).

Figura 2 - Riqueza total de coleópteros coletados com armadilha de interceptação de voo, em diferentes alturas, em povoamento de $P$. taeda, entre novembro de 2011 e maio de 2012, no município de Santa Maria/RS.

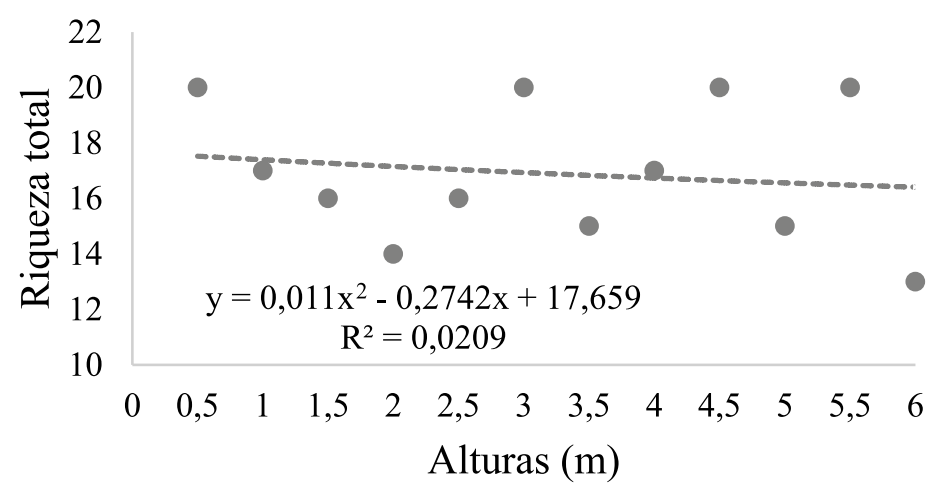

A distribuição aleatória da coleopterofauna, tanto em relação a abundância quanto para diversidade, pode estar associada a grande diversidade de espécies coletadas. Neste contexto, conforme pode ser observado na Tabela 1 , mesmo a espécie mais abundante teve apenas 42 exemplares amostrados, o que representa somente $7,37 \%$ do total de indivíduos coletados (570 exemplares). Assim, a grande diversidade de espécies e a baixa dominância pode ter dificultado a identificação de um padrão entre a altura de voo, abundância e a diversidade.

\section{Altura de voo (distribuição vertical) dos principais táxons (gêneros e espécies mais abundantes)}

Para análise da distribuição vertical foram consideradas as espécies mais abundantes, correspondendo a Corinthiscus sp.1, Domoptolis menestriesii, Euetheola humilis, Neoclytus pusillus, Temnoscheila sp.2, Tenebroides sp.1. e Tenebroides sp. 2 .

O gênero Corinthiscus apresentou 25 espécimes distribuídos em duas espécies. Corinthiscus sp. 1 obteve a maior abundância (23 exemplares), com a altura de 1,5 $\mathrm{m}$ se destacando com oito espécimes coletados (Figura 3). Porém, não foi possível determinar um comportamento em relação à altura de voo, ou seja, não foi identificado um padrão que relacione a altura de voo com a respectiva abundância da espécie. O gênero Corinthiscus não apresentou altura preferencial de voo pois a equação gerada apresentou um baixo coeficiente de determinação $\left(\mathrm{R}^{2}=0,0781\right)$.

A espécie Domoptolis menestriesii obteve 10 exemplares durante o período de coleta, sendo que nas 
Distribuição vertical e diversidade de coleópteros em povoamento de Pinus taeda L. (Pinaceae)

alturas de 4,0, 4,5, 5,0 e 5,5 m, foram observados dois indivíduos em cada (Figura 3). Verificou-se que a faixa preferencial de voo de $D$. menestriesii está 4,0 e 5,5 m, dado importante para o monitoramento desta espécie em cultivos de $P$. taeda.

Figura 3 - Distribuição vertical das espécies mais abundantes coletadas com armadilha de interceptação de voo, em diferentes alturas, em povoamento de $P$. taeda, entre novembro de 2011 e maio de 2012, no município de Santa Maria/RS.
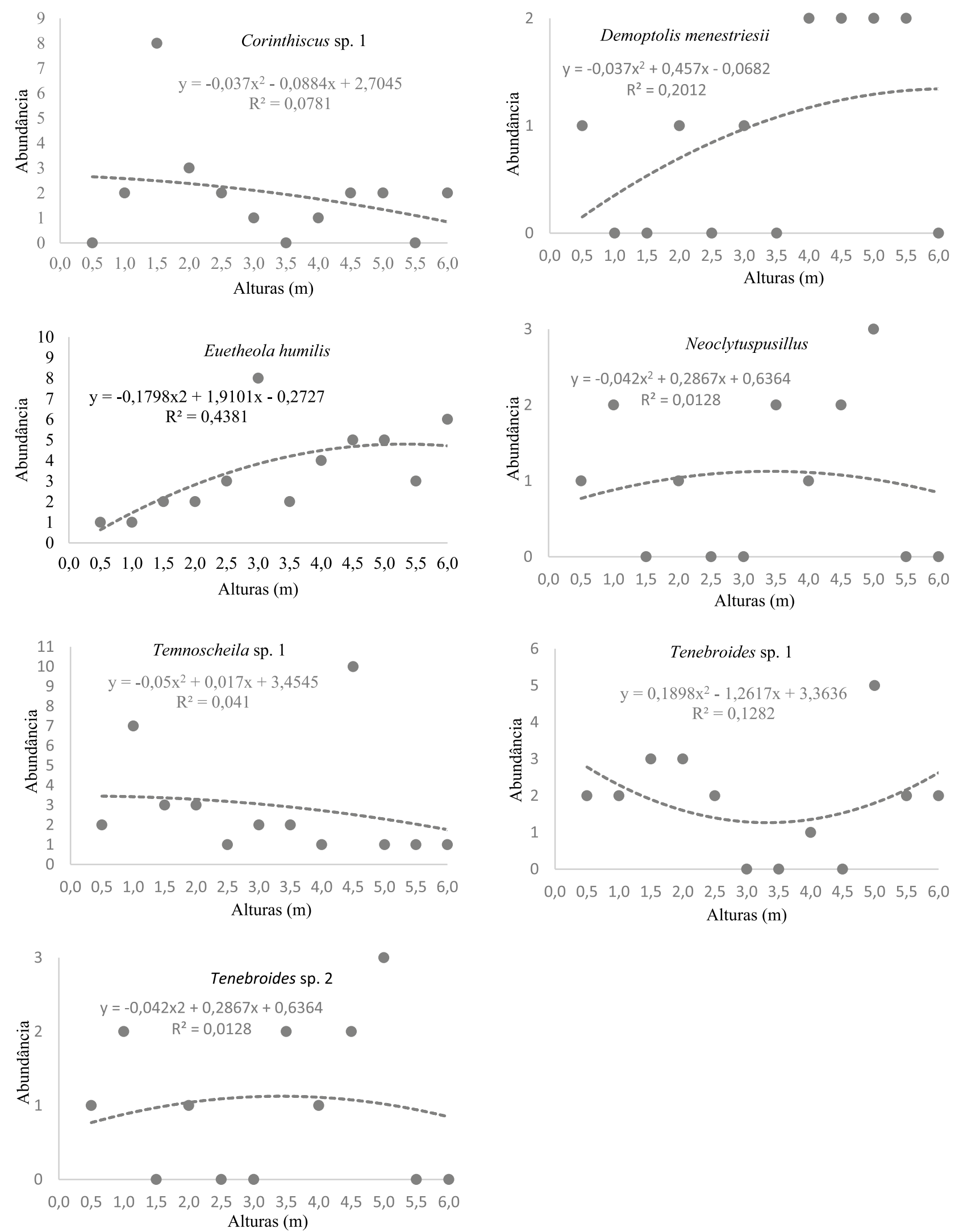

Cad. Ciênc. Agrá., v. 12, p. 01-11, https://doi.org/10.35699/2447-6218.2020.21654 
A espécie E. humilis destacou-se com 42 espécimes coletados, representando 7,37\% do total da coleopterofauna no povoamento de $P$. taeda. Corroborando com o resultado obtido nesse trabalho, Bernardi et al. (2010) identificou E. humilis como espécie mais abundante, representando $21,5 \%$ dos coleópteros coletados em plantio de Eucalyptus spp., na região sul do Rio Grande do Sul. É importante destacar ainda que, E. humilis apresentou distribuição em todas as alturas avaliadas nesse trabalho, com destaque para 3,0, 4,5, 5,0 e 6,0 m, conforme é apresentado na Figura 3. Assim, embora com um baixo coeficiente de determinação $\left(\mathrm{R}^{2}=0,4381\right)$, a abundância desta espécie tende a ser um pouco maior com a elevação da altura.

Neoclytus pusillus teve 12 indivíduos coletados e não se verificou preferência por nenhuma das alturas estudadas $\left(R^{2}=0,0128\right)$, conforme pode ser observado na Figura 3. Temnoscheila sp.2 foi encontrada em todas as alturas analisadas, destacando a altura de $4,5 \mathrm{~m}$, com 10 espécimes (Figura 4). Porém, não foi possível determinar um comportamento em relação a altura de voo, ou seja, não foi identificado um padrão que relacione a altura de voo com a respectiva abundância da espécie $\left(R^{2}=0,0410\right)$.

Com relação ao gênero Tenebroides, foram encontrados 45 espécimes, distribuídos em três espécies. A espécie Tenebroides sp. 1 apresentou abundância de 22 indivíduos, com destaque para a altura de $5,0 \mathrm{~m}$, com dez espécimes coletados (Figura 3). Porém, não foi possível determinar um comportamento em relação à altura de voo, ou seja, não foi identificado um padrão que relacione a altura de voo com a respectiva abundância da espécie $\left(\mathrm{R}^{2}=0,1282\right)$.
Assim, conforme se observa na Figura 3, apenas E. humilis apresentou uma relação entre a altura de voo e a abundância $\left(R^{2}=0,4381\right)$, assim pelo coeficiente de determinação identificado, existe uma baixa relação entre a altura de instalação das armadilhas e a abundância, nos quais a captura de $E$. humilis tende a ser maior quanto maior a altura de instalação da armadilha.

Para as demais espécies mais abundantes não foi encontrada uma relação entre altura de voo e abundância. Tal fato pode estar relacionado ao fato de insetos, especificamente os da Ordem Coleoptera, terem elevada abundância de espécies, com preferências diferentes, não sendo possível identificar nesse estudo um padrão de voo comum entre elas.

\section{Conclusão}

As famílias Cerambycidae, Curculionidae, Trogossitidae e Scarabaeidae foram as mais abundantes e não possuem preferência por uma única altura de voo, sendo encontradas em todas as alturas da armadilha de interceptação. A abundância total de coleópteros não varia entre as alturas de $0,5 \mathrm{~m}$ a 6,0 em povoamento de Pinus taeda na região central do Estado do Rio Grande do Sul.

\section{Agradecimentos}

Os autores agradecem ao Dr. Pedro Giovâni da Silva, pesquisador do Laboratório de Ecologia de Insetos, Instituto de Ciências Biológicas da Universidade Federal de Minas Gerais, pela identificação das espécies de coleópteros.

\section{Referências}

Alvares, C. A.; Sentelhas, P. C.; Stape, J. L. 2017. Modeling monthly meteorological and agronomic frost days, based on minimum air temperature, in Center-Southern Brazil. Theor Appl Climatol 134, 177-19. Doi: https://doi.org/10.1007/s00704-017-2267-6.

Bernardi, O.; Garcia, M. S.; Cunha, U. S.; Back, E. C. U.; Bernardi, D.; Ramiro, A. R.; Finkenauer, E. 2008. Ocorrência de Euetheola humilis (Burmeister) (Coleoptera: Scarabaeidae) em Eucalyptus saligna Smith (Myrtaceae), no Rio Grande do Sul. Neotropical entomology. v. 37 n.1. Doi: http://dx.doi.org/10.1590/S1519-566X2008000100017.

Bernardi, O.; Garcia, M. S.; Silva, E. J. E.; Zazycki, L. C. F.; Bernardi, D.; Miorelli, D.; Ramiro, G. A.; Finkenauer, E. 2010. Coleópteros coletados com armadilhas luminosas e etanólicas em plantio de Eucalyptus spp. no sul do Rio Grande do Sul. Ciência Florestal, v. 20, n. 4, p. 579 - 588. Disponível em: https://periodicos.ufsm.br/cienciaflorestal/ article/view/2416.

Bertim, V. M. Flutuação populacional de degradadores da madeira em função da altura de coleta. 2013. Seropédica: Universidade Federal Rural do Rio de Janeiro, 28 p. Monografia Bacharel em Engenharia Florestal. Disponível em: http://www.bibliotecaflorestal.ufv.br/ handle/123456789/13986.
Carvalho, A. G.; Trevisan, H. Novo Modelo de Armadilha para Captura de Scolytinae e Platypodinae (Insecta, Coleoptera) Floresta e Ambiente, v. 22, n. 4, p. 575-578, 2015.

Dorval, A.; Peres Filho, O; Marques, E. N.; Moura, R. G. Coleópteros em plantios de Eucalyptus citriodora e E. urophylla em Cuiabá, estado de Mato Grosso. 2007. Revista da Agricultura. V. 82, n. 3. Doi: https:// doi.org/10.37856/bja.v82i3.1460.

Flechtmann, Carlos \& H.T.Z, Couto \& C.L, Gaspareto \&, Evoneo. 1995 Scolytidae em Reflorestamento com Pinheiros Tropicais. Piracicaba: IPEF, 1995. 201 p. Disponível em: https://www.ipef.br/publicacoes/ manuais/manual_pragas_v4.pdf.

Freitas, F. A.; Zanuncio, T. V.; Lacerda, M. C.; Zanuncio, J. C. 2002. Fauna de coleoptera coletada com armadilhas luminosas em plantio de Eucalyptus grandis em Santa Bárbara, Minas Gerais. Rev. Árvore. vol.26, n.4, pp.505-511. Doi: https://doi.org/10.1590/S010067622002000400014. 
Distribuição vertical e diversidade de coleópteros em povoamento de Pinus taeda L. (Pinaceae)

Ganho, N. G.; Marinoni, R. C. 2006. A variabilidade espacial das famílias de Coleoptera (Insecta) entre fragmentos de Floresta Ombrófila Mista Montana (Bioma Araucária) e plantação de Pinus elliottii Engelmann, no Parque Ecológico Vivat Floresta, Tijucas do Sul, Paraná, Brasil. Rev. Bras. Zool. Curitiba, v.23, n.4. Doi: http://dx.doi.org/10.1590/ S0101-81752006000400024.

Hammer, O.; Harper, D. A. T.; Ryan, P. D. 2001. PAST: Paleontological statistics software package for education and data analysis. Palaeontologia Electronica, v. 4, n. 1, 9 p. Disponível em: https://palaeo-electronica. org/2001_1/past/past.pdf.

IBA - INDÚSTRIA BRASILEIRA DE ÁRVORES. 2019. Relatório 2019. 80 p., 2019.

LEMES, P. G.; ANJOS, N. dos.; CORDEIRO, G. Injúrias e oviposição de Oncideres impluviata (Germar) (Col.: Cerambycidae) em Piptadenia gonoacantha (Mart.) Macbr. Comunicata Scientiae, Bom Jesus, v. 2, n. 1, p. 53-56, 2011. Disponível em: https://www.researchgate. net/publication/267034296_Injurias_e_oviposicao_de_Oncideres impluviata_Germar_Col_Cerambycidae_em_Piptadenia_gonoacantha Mart_Macbr.

Machado, L. M. 2013. Determinação da altura de voo de escolitídeos em mata nativa e em povoamento de Pinus taeda. 56 p. Santa Maria: Universidade Federal de Santa Maria. Dissertação Mestrado. Disponível em: https://repositorio.ufsm.br/bitstream/handle/1/8709/ MACHADO,\%20LEONARDO\%20MORTARI.pdf.

Machado, L. M.; Costa, E. C. 2017. Altura de voo de escolitíneos (Coleoptera, Scolytinae) em povoamento de Pinus taeda L. no Sul do Brasil. Ciência Florestal, v. 27, n. 2, p. 669-678. Doi: http://dx.doi. org/10.5902/1980509827751.
Morón, M. A. 2004. Melolontídeos edafícolas. In: SALVADORI, J. R.; ÁVILA, C. J.; SILVA, M. T. B (Eds). Pragas de solo no Brasil. Passo Fundo: Embrapa Trigo. Dourados: Embrapa Agropecuária Oeste. Cruz Alta: Fundacep Fecotrigo, p.133- 166.

Murari, A. B.; Costa, E. C.; Boscardin, J.; Garlet, J. 2012. Modelo de armadilha etanólica de interceptação de voo para captura de escolitíneos (Curculionidae: Scolytinae). Pesquisa Florestal Brasileira, Colombo, v. 32, n. 69, p. 115-117. Doi: 10.4336/2012.pfb.32.69.115

Peres Filho, O.; Dorval, A.; Berti Filho, E. 2006. A entomofauna associada à Teca, Tectona grandis Linn. f. (Verbenaceae), no estado de Mato Grosso. Piracicaba: IPEF, 58 p.

Peres Filho, O.; Barbosa, J. I.; Souza, M. D.; Dorval, A. 2012. Altura de voo de bostriquídeos (Coleoptera: Bostrichidae) coletados em Floresta Tropical Semidecídua, Mato Grosso. Pesquisa Florestal Brasileira, Colombo, v. 32, n. 69, p. 101-107, 2012. Doi: 10.4336/2012. pfb.32.69.101.

Pinto, R.; Junior, J. S. Z.; Zanuncio, J. C.; Barbosa, M. C. 2004. Coleópteros coletados com armadilhas luminosas em plantio de Eucalyptus urophylla na região Amazônica Brasileira. Ciência Florestal, Santa Maria, v. 14, n. 1. Doi: https://doi.org/10.5902/198050981787.

Zanuncio, J. C.; Bragança, M. A. L.; Laranjeiro, A. J.; Fagundes, M. 1993. Coleópteros associados a eucaliptocultura nas regiões de São Mateus e Aracruz, Espirito Santo. Ceres, Vicosa, v. 41, n. 22, p. 584-90. Disponível em: http://www.ceres.ufv.br/ojs/index.php/ceres/article/view/2230. 